\title{
Estimating rainfall-runoff model parameters using the iterative ensemble smoother
}

\author{
F.R. Bennett $^{a}{ }^{(1)}$ and J. Doherty ${ }^{b}$ \\ ${ }^{a}$ Department of Environment and Science, Queensland Government, Australia \\ ${ }^{b}$ Watermark Numerical Computing, Brisbane, Australia \\ Email: frederick.bennett@des.qld.gov.au
}

\begin{abstract}
Catchment water quality models are an important tool for understanding the impacts of land management practice on the water quality of the receiving waters of the Great Barrier Reef lagoon. As part of the Paddock to Reef program the Great Barrier Reef Catchment Loads Modelling Program estimates average annual loads of key pollutants (sediment, nutrients and pesticides) for each of the 35 catchments draining to the Great Barrier Reef. Since catchment models assume that constituent generation and transport within the catchment is largely controlled by rainfall and runoff, it is imperative that the hydrology calibration approach underpinning the catchment model is rigorous and achieves the best possible results. Because catchment models are conceptual representations of very complex landscape systems any forecasts and predictions they produce will be subject to uncertainty and quantifying uncertainty is an important aspect of analysing model performance.
\end{abstract}

Various methods derived from a range of statistical frameworks have been applied to study uncertainty of rainfall-runoff models. Perhaps the most intuitive way of approaching uncertainty analysis is via the formalism of Bayes' theorem where some prior understanding of the model parameters is updated once exposed to relevant data. As elegant as Bayesian uncertainty analysis may be, there are practical limitations to implementing it. The equations defining Bayes' theorem often have no analytic solution, or at least one that is tractable, one must resort to numerical methods to complete the process. In practice, this usually involves a campaign of stochastically sampling from the Bayesian posterior distribution to construct a statistical facsimile. This can be a computationally exhausting process, particularly when an expensive model is used, the prior is significantly divergent from the posterior and a large number of parameters is involved.

Ensemble methods such as the iterative ensemble smoother (IES) have been developed to alleviate much of the computational overhead demanded by the uncertainty quantification of environmental models, particularly those that involve high dimensional parameter spaces. On the face of it, the IES would seem to fit very well with the problem presented by catchment water quality models but to date, there is very little evidence of this. In this study, we apply a Gauss-Levenberg-Marquardt form of the IES to the calibration and uncertainty analysis of a rainfall-runoff model. The IES is found to be an efficient and powerful method for conditioning model parameters and providing robust uncertainty estimates adhering to the spirit of Bayesian statistics.

Keywords: Ensemble smoothers, Bayesian methods, uncertainty analysis 


\section{INTRODUCTION}

The use of the Iterative Ensemble Smoother (IES) (Evensen, 2018) for solving inverse problems encountered in the fields of reservoir-engineering and groundwater modelling is well established (Oliver et al., 2008). The veritable symphony of available literature covering this topic does not however prepare one for the sound of crickets accompanying a search for reports on IES applications in the field of surface water modelling.

Surface water models, such as conceptual rainfall-runoff (CRR) models can be computationally expensive and estimating appropriate parameter values may be a complex undertaking. For reliable decision making, CRR model parameter uncertainties are also required. Within the Bayesian paradigm, parameter uncertainties can be estimated by stochastically sampling the posterior distribution using Markov-chain Monte-Carlo (MCMC) methods for example. The computational expense of this type of sampling may be prohibitive depending on the complexity of the model and the dimensionality of the model parameter set. Although sampling efficiency can be significantly improved through the use of sequential Monte-Carlo sampling or the Metropolis-Hastings algorithm, the number of model evaluations required to satisfactorily populate the posterior density can still render the approach impractical for all intents and purposes.

Compared to the more stochastically driven approaches, Ensemble based methods have a major advantage in that they require much fewer model evaluations, particularly for higher dimension problems. Ensemble methods are theoretically grounded in Bayesian statistics and commence by drawing an ensemble of model parameters from a multivariate prior distribution. Informed by the available data, each realization from the parameter prior is then maximum a posteriori conditioned to give a sample from the posterior distribution. The ensemble smoother (ES) (Leeuwen, 2001) is a modification of the sequential ensemble Kalman filter (EnKF) (Evensen, 1994). Although the Kalman filter and the Ensemble Smoother are closely related mathematically, there are important distinctions in their emphasis. Where the Kalman filter seeks to recursively estimate states and/or parameters, assimilating information one step at a time, the ensemble smoother works in "batch" mode, running the model over the entirety of its simulation time. This latter methodology is much more typical of the approach generally employed for rainfall-runoff model parameter calibration studies. The IES improved upon the ES by updating the parameters in a number of finite incremental steps which served to address problems associated with non-linear response of the forward solver.

Several algorithms have been proposed controlling how the IES updates parameters at each iteration (Evensen, 2018). In this study, we investigate the use of the Levenberg-Marquardt ensemble randomized maximum likelihood (LM-EnRML) algorithm of Chen and Oliver (Chen and Oliver, 2013) for assimilating streamflow data in a CRR model. The Levenberg-Marquardt method regularizes the direction and length of the parameter update step. Problems that may be encountered due to highly nonlinear responses to parameter movement can be avoided by including a step damping parameter, particularly during the early iterations.

\section{STUDY AREA - CATTLE CREEK CATCHMENT}

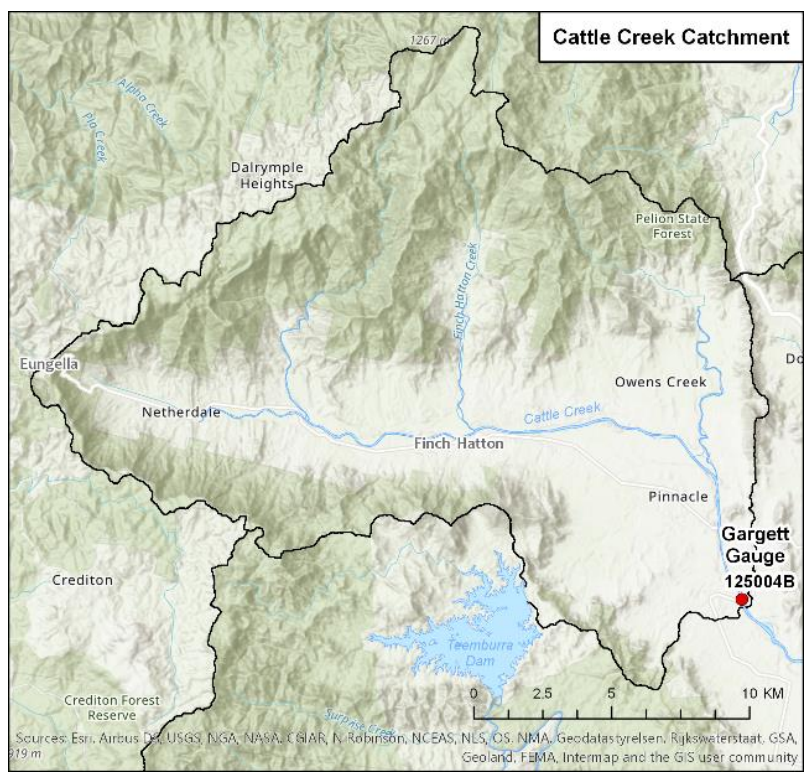

Figure 1. Map of the Cattle Creek catchment showing the Gargett flow observation point 125004B
The Mackay Whitsunday NRM region covers about $9,130 \mathrm{~km}^{2}$ and comprises the Pioneer, Proserpine and O'Connell Rivers, and Plane Creek basins draining to the Great Barrier Reef lagoon. The Whitsunday Islands group is situated off the coast between Bowen and Mackay. Upper Cattle Creek is a tributary of the Pioneer River located in the west of the Mackay Whitsunday region. The catchment area receives high annual rainfall in excess of $3000 \mathrm{~mm}$. The highlands in the headwaters of Upper Cattle Creek catchment are heavily timbered with rainforest while the lower reaches are dominated by open woodland. Eungulla protects over $70 \%$ of the catchment area on these steep upper slopes. On the alluvial floodplains $14 \%$ of the catchment is under cane production, and $13 \%$ under grazing. Downstream, Mirani Weir creates a barrier to fish migration for the Upper Cattle Creek flow. 


\section{METHODOLOGY}

\subsection{The iterative ensemble smoother}

The problem being considered is the estimation of an unknown set of model parameters represented by the vector $m \in \mathbb{R}^{N_{m}}$ given observational data $d_{o b s} \in \mathbb{R}^{N_{d}}$. Assuming a non-linear model, $g, d_{o b s}$ can be estimated by

$$
d_{o b s}=g(m)+\epsilon
$$

where $\epsilon$ is a random error from which the likelihood $p\left(d_{o b s} \mid g(m)\right)$ arises.

Within the Bayesian framework, the conditional posterior probability density function (PDF) is related to the likelihood and prior PDFs through Bayes theorem

$$
p\left(m \mid d_{o b s}\right) \propto p\left(d_{o b s} \mid g(m)\right) p(m)
$$

where $p(m)$ is the Bayesian prior PDF of $m$. If the assumption that the observation errors are normally distributed is imposed then

$$
p\left(d_{o b s} \mid g(m)\right) \propto \exp \left[-\frac{1}{2}\left(d_{o b s}-g(m)\right)^{T} C_{D}^{-1}\left(d_{o b s}-g(m)\right)\right]
$$

where $C_{d}$ is the covariance matrix of the observation errors. Likewise, if the prior distribution is also Gaussian then

$$
p(m) \propto \exp \left[-\frac{1}{2}\left(\mathrm{~m}-m_{p r}\right)^{T} C_{m}^{-1}\left(\mathrm{~m}-m_{p r}\right)\right]
$$

Where $m_{p r}$ is the prior parameter estimate and $C_{m}$ is the covariance matrix of the prior distribution.

If unconditional realisations of the parameter prior, $m_{u}$, and the data likelihood, $d_{u}$, are drawn from their respective PDFs, it is straight forward to show using Equations 3 and 4 that an estimate of the maximum a posteriori (MAP) can be found by minimizing the objective function

$$
O(m)=\left(d_{u}-g(m)\right)^{T} C_{D}^{-1}\left(d_{u}-g(m)\right)+\left(m-m_{u}\right)^{T} C_{M}^{-1}\left(m-m_{u}\right) .
$$

which generates an approximate sample from the conditioned posterior.

The option for minimizing the objective function in Equation 5 that will be investigated here is the regularised Gauss-Levenberg-Marquardt algorithm (GLM) with an iterative parameter improvement given by:

$$
m_{l+1}=m_{l}-\left[\left(1-\lambda_{l}\right) C_{M}^{-1}+J_{l}^{T} C_{D}^{-1} J_{l}\right]^{-1}\left[C_{M}^{-1}\left(m_{l}-m_{u}\right)+J_{l}^{T} C_{D}^{-1}\left(g\left(m_{l}\right)-d_{u}\right)\right]
$$

where $m_{l}$ is the parameter vector from the $l^{\text {th }}$ iteration, $J_{l}$ is the Jacobian matrix of $g$ evaluated at $m_{l}$ and the scalar $\lambda_{l}$ is the "Marquardt lambda" that assists in accommodating model nonlinearity in the inversion process.

A population of samples from the posterior PDF can now be generated by repeatedly calibrating unconditional realisations using the MAP form of the GLM in Equation 6.

It is obvious however that the major computational burden for this method of sampling from the posterior is the filling of the Jacobian matrix at each GLM iteration, particularly when numerical derivatives are used, and the generative model is computationally expensive. The number of model evaluations required to fill the Jacobian scales linearly with the number of parameters being optimised. As $O(m)$ needs to be minimised for each realisation, the effort involved in drawing a satisfactory number samples from the conditioned posterior can render this method wildly impractical for all intents and purposes.

In order to address this burden, Chen and Oliver have proposed an empirical approximation to the Jacobian using an iterative ensemble smoother (IES) that can substantially reduce the computational effort. The resulting ensemble GLM is 
Bennett and Doherty, Estimating rainfall-runoff model parameters using the iterative ensemble smoother

$$
\begin{gathered}
m_{l+1}^{e}=m_{l}^{e}-\left[\left(1-\lambda_{l}\right) C_{M}^{-1}+J_{e m p, l}^{T} C_{D}^{-1} J_{e m p, l}\right]^{-1}\left[C_{M}^{-1}\left(m_{l}^{e}-m_{u}^{e}\right)\right. \\
\left.+J_{e m p, l}^{T} C_{D}^{-1}\left(g\left(m_{l}^{e}\right)-d_{u}^{e}\right)\right]
\end{gathered}
$$

where, for an ensemble of $N_{e}$ realisations, $m_{l}^{e}$ and $m_{u}^{e}$ are the current and unconditioned parameter ensembles $\left(N_{e} \times N_{m}\right), d_{u}^{e}$ is the initial observation ensemble $\left(N_{e} \times N_{d}\right)$ and $g\left(m_{l}^{e}\right)$ are the modelled data evaluated at $m_{l}^{e}$. The empirical ensemble Jacobian can now be found by solving the equation

$$
\Delta d_{l}^{e} \approx J_{e m p, l} \Delta m_{l}^{e}
$$

giving

$$
J_{e m p, l} \approx C_{D}^{1 / 2} \Delta d_{l}^{e}\left(\Delta m_{l}^{e}\right)^{-1} C_{M}^{-1 / 2}
$$

where

$$
\Delta d_{l}^{e}=C_{D}^{-1 / 2}\left(g\left(m_{l}^{e}\right)-\overline{g\left(m_{l}^{e}\right)}\right) / \sqrt{N_{e}-1}
$$

and

$$
\Delta m_{l}^{e}=C_{M}^{-1 / 2}\left(m_{l}^{e}-\overline{m_{l}^{e}}\right) / \sqrt{N_{e}-1}
$$

With this formulation of the IES, the model only has to be evaluated once for each member of the ensemble to construct the empirical Jacobian which can substantially reduce the computational burden, especially when calibrating a large number of parameters.

\begin{tabular}{|c|c|c|}
\hline Parameter & Description & Range \\
\hline UZTWM & Upper Zone Tension Water Storage Maximum (mm) & $12-180$ \\
\hline UZFWM & Upper Zone Free Water Storage Maximum (mm) & $5-155$ \\
\hline UZK & Upper Zone Lateral Drainage Rate & $0.1-1.0$ \\
\hline ZPERC & The potential increase in percolation from saturated to dry conditions & $1.0-600$ \\
\hline REXP & Exponent in Percolation Relationship & $1.0-10.0$ \\
\hline PCTIM & Permanently impervious fraction of the basin & $1.0 \mathrm{E}-5-0.25$ \\
\hline SARVA & Fraction of the basin covered by streams, lakes, and riparian vegetation & $1.0 \mathrm{E}-5-0.11$ \\
\hline SSOUT & Subsurface outflow along the channel (mm/day) & $1.0 \mathrm{E}-5-0.11$ \\
\hline ADIMP & Fraction of the basin becomes impervious as all tension water are met & $1.0 \mathrm{E}-5-0.15$ \\
\hline PFREE & Proportion of percolated water directly enters Free Water Storage & $1.0 \mathrm{E}-2-0.5$ \\
\hline LZTWM & Lower Zone Tension Water Storage Maximum (mm) & $1.0 \mathrm{E}-10-600$ \\
\hline LZFSM & Lower Zone Free Water Supplementary Storage Maximum (mm) & $1.0-350$ \\
\hline LZFPM & Lower Zone Free Water Primary Storage Maximum (mm) & $1.0-600$ \\
\hline LZSK & Lower Zone Supplementary Drainage Rate & $1.0 \mathrm{E}-3-0.9$ \\
\hline LZPK & Lower Zone Primary Drainage Rate & $1.0 \mathrm{E}-3-0.6$ \\
\hline RSERVE & Fraction of lower zone free water unavailable for transpiration & $0.2-0.4$ \\
\hline SIDE & The ratio of non-channel baseflow (deep recharge) to channel (visible) baseflow. & $1.0 \mathrm{E}-5-0.1$ \\
\hline ROUTC & Regional constant calculated from reach length & $1.0-1.0 \mathrm{E} 6$ \\
\hline ROUTP & Routing parameter power & $0.6-0.1$ \\
\hline
\end{tabular}

The present study employs the pest-ies tool which is a model independent implementation of GLM-IES (White, 2018).

\subsection{Rainfall runoff model}

Table 1. Parameter descriptions and ranges used in the Sacramento model and refined in the assimilation process 


\section{RESULTS AND DISCUSSION}

Results from the IES analysis are based on ensemble composed of 500 data realisations and corresponding parameters. The final posterior distributions of the IES parameters are shown in Figure 2. Most parameters appear to be well identified with symmetrical distributions about the mean. The UZTWM distribution is clipped at higher values and it may be interesting to investigate the effects of increasing the upper bound on the allowed parameter range.
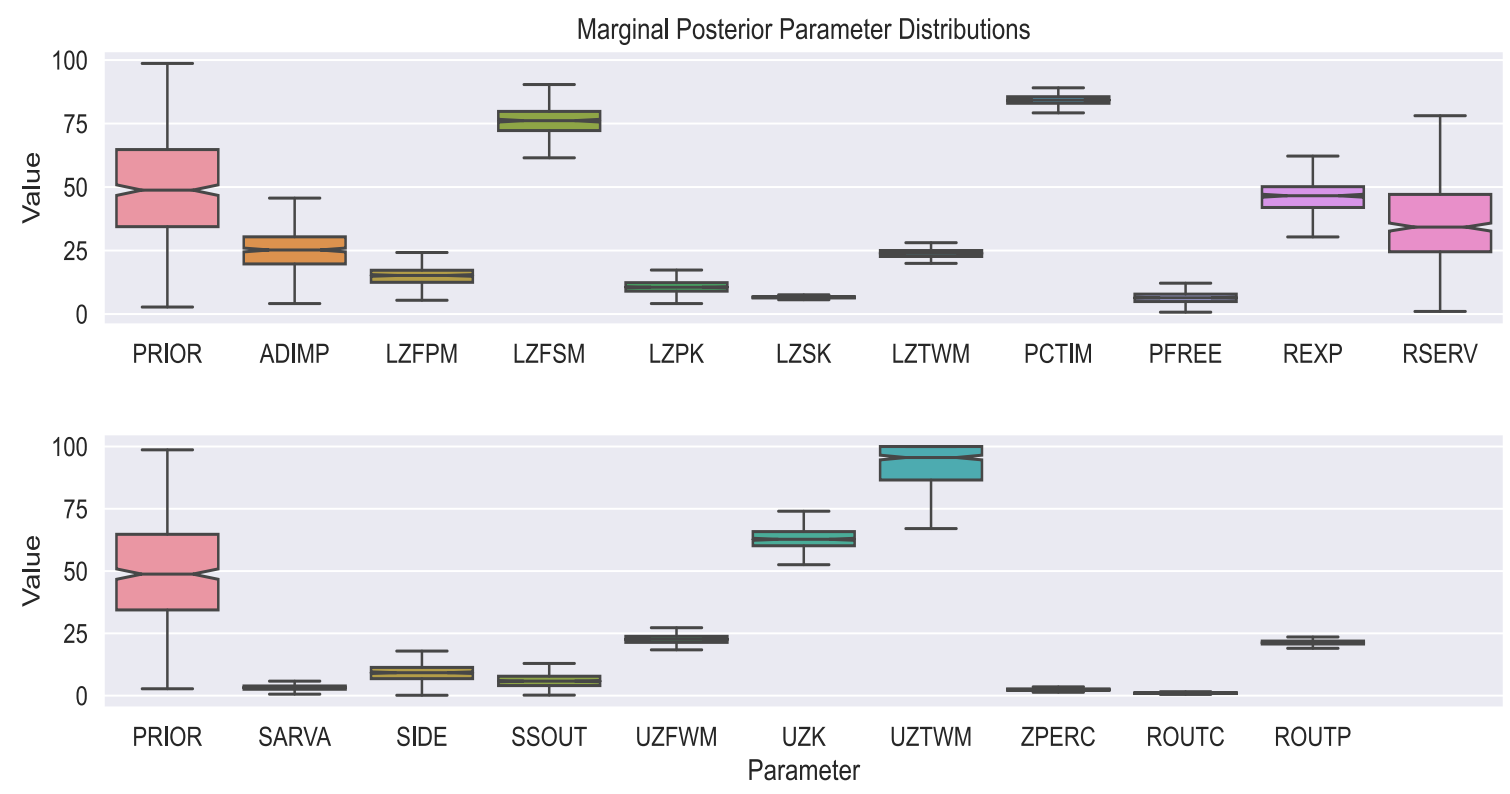

Figure 2. Marginal posterior parameter distributions. For convenience, the parameter ranges from Table 1 have been transformed to span a uniform range of 0-100. The results are presented in the upper and lower panels of the graphic which both include the same prior distribution for comparison.

Figure 3 shows a short, extracted flow timeseries comparison between the observed streamflow data and the median flow value from the final IES data ensemble. The observed and simulated stream flows are in good agreement generally depicting satisfactory flow magnitudes and flow rise and fall characteristics.

Conventional quantitative statistics can be used to understand the performance of the ensemble. Table 2 summarises the distribution of the Nash-Sutcliffe efficiency coefficient (NSE) (Nash and Sutcliffe, 1970), $r^{2}$ and PBIAS for simulated ensemble members and paired data noise realisations. The mean NSE of 0.882 with a small standard deviation of 0.002 based on 500 samples suggests that all ensemble realisations are performing very well with no significant deviations. The PBIAS spread is interesting with a standard deviation of $0.43 \%$ around a mean of $3.38 \%$ hinting that there may be a systematic tendency to overestimate the aggregated discharge volume independently of starting parameters and data perturbation. Considering that all elements of the ensemble match their corresponding data realisation with a PBIAS $<5.0 \%$ is still a pleasing result suggesting that the Sacramento model is skilfully able to predict stream flows satisfactory.
Table 2. mean and standard deviation of model performance statistics aggregated over the ensemble

\begin{tabular}{|l|r|r|}
\hline & $\mu$ & $\sigma$ \\
\hline$N S E$ & 0.882 & 0.002 \\
\hline$r^{2}$ & 0.883 & 0.002 \\
\hline PBIAS & $3.38 \%$ & $0.43 \%$ \\
\hline
\end{tabular}




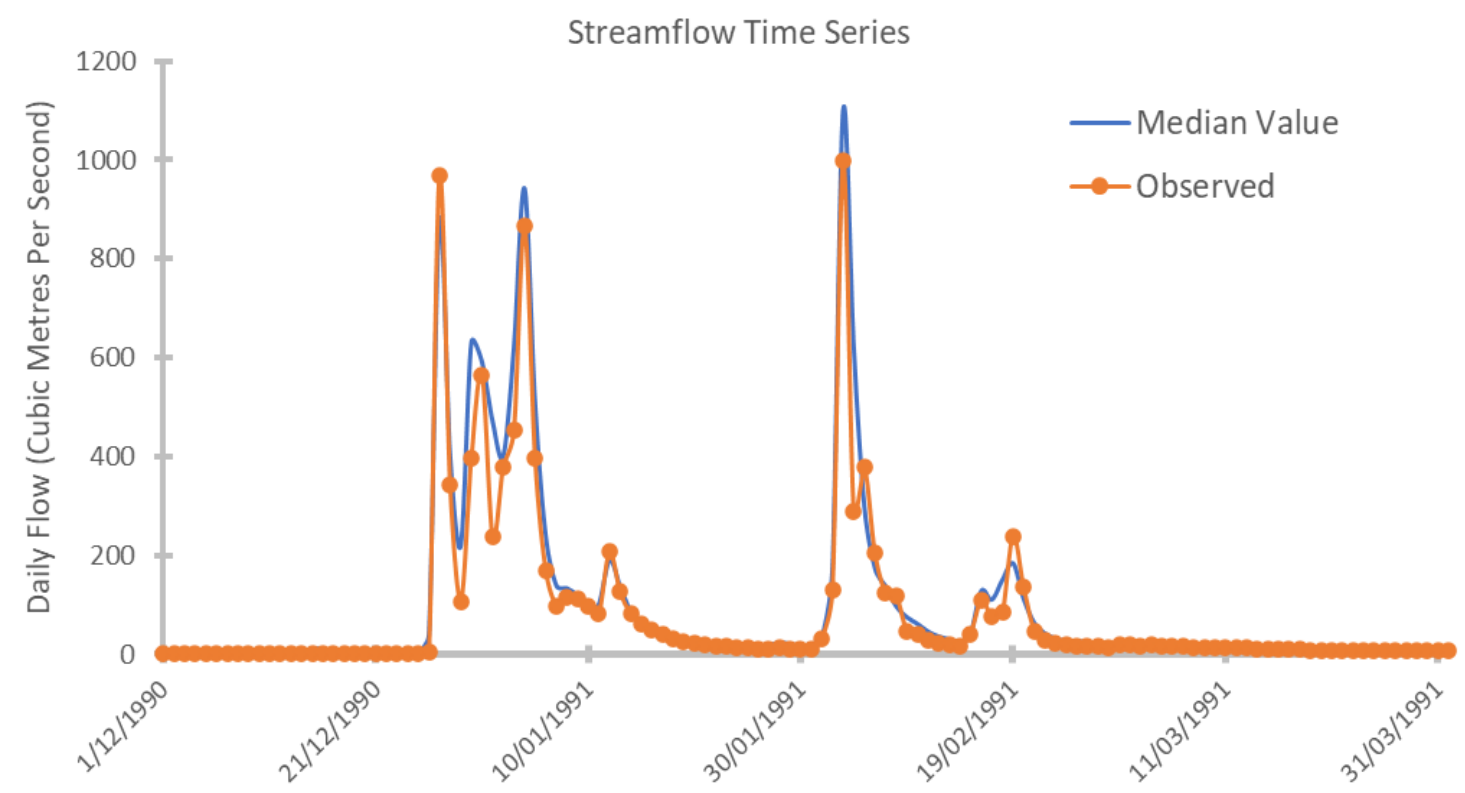

Figure 3. Comparison of predicted daily flow with observed daily flow at gauging station

\section{CONCLUSIONS}

This study investigated the use of the (LM-EnRML) form of the IES for estimating Sacramento CRR model parameter uncertainty for the Cattle Creek catchment. An ensemble of 500 prior parameter sets were simultaneously assimilated to corresponding data realisations drawn from a noise distribution placed around the observed streamflow data. Following optimization, an analysis of the performance of each individual ensemble member showed a very narrow range of performance statistic values (NSE, $\mathrm{r}^{2}$ and \% bias) suggesting that the posterior ensemble was well balanced with no anomalous behaviour. Almost all Sacramento parameters were well identified apart from RSERVE where the marginal posterior distribution deviated only slightly from the prior and UZTWM which was discussed in the previous section.

Even though this study presents a single case study, experience in our group suggests that the IES appears to be a powerful tool for uncertainty analysis of CRR models. Where the distribution of data measurement errors can be satisfactorily represented by a multivariate normal distribution, the IES should yield a good estimation of the Bayesian posterior when a gaussian prior is placed on the parameters. Although several flavours of the IES exist, the Levenberg-Marquardt form should be among the most robust when it comes to handling nonlinearities on the model response function hypersurface.

In the case where the model inversion problem is ill-posed and the data has a degree of Gaussian uncertainty, the formulation of the IES is such that a valid Bayesian posterior should result. This is strictly true in the case of an exact model and linear response function and is approximate in the nonlinear case (Evensen, 2018). When model structural error begins to dominate and surpasses the magnitude of the data uncertainty, the IES will begin to suffer the same problems as any method that doesn't account for model deficiencies such as systematically biased parameter estimates. Investigations into how to account for more epistemic sources of uncertainty is the subject of ongoing work (Vilas et al., 2021).

\section{REFERENCES}

Burnash, R.J.C., Ferral, R.L., McGuire, R.A., 1973. A generalized streamflow simulation system: conceptual modeling for digital computers. U. S. Dept. of Commerce, National Weather Service, Sacramento, Calif.

Chen, Y., Oliver, D.S., 2013. Levenberg-Marquardt forms of the iterative ensemble smoother for efficient history matching and uncertainty quantification. Comput. Geosci. 17, 689-703. https://doi.org/10.1007/s10596-013-9351-5

Evensen, G., 2018. Analysis of iterative ensemble smoothers for solving inverse problems. Comput. Geosci. 22, 885-908. https://doi.org/10.1007/s10596-018-9731-y 
Evensen, G., 1994. Sequential data assimilation with a nonlinear quasi-geostrophic model using Monte Carlo methods to forecast error statistics. J. Geophys. Res. Oceans 99, 10143-10162. https://doi.org/10.1029/94JC00572

Jeffrey, S.J., Carter, J.O., Moodie, K.B., Beswick, A.R., 2001. Using spatial interpolation to construct a comprehensive archive of Australian climate data. Environ. Model. Softw. 16, 309-330. https://doi.org/10.1016/S1364-8152(01)00008-1

Leeuwen, P.J. van, 2001. An Ensemble Smoother with Error Estimates. Mon. Weather Rev. 129, $709-728$. https://doi.org/10.1175/1520-0493(2001)129<0709:AESWEE>2.0.CO;2

Nash, J.E., Sutcliffe, J.V., 1970. River flow forecasting through conceptual models part I - A discussion of principles. J. Hydrol. 10, 282-290. https://doi.org/10.1016/0022-1694(70)90255-6

Oliver, D.S., Reynolds, A.C., Liu, N., 2008. Inverse Theory for Petroleum Reservoir Characterization and History Matching. Cambridge University Press, Leiden.

Vilas, M.P., Bennett, F.R., Verburg, K., Adams, M.P., 2021. Considering unknown uncertainty in imperfect models: nitrogen mineralization as a case study, in: MODSIM2021, 24th International Congress on Modelling and Simulation. Presented at the 23rd International Congress on Modelling and Simulation (MODSIM2019), Modelling and Simulation Society of Australia and New Zealand, Sydney, p. 7.

White, J.T., 2018. A model-independent iterative ensemble smoother for efficient history-matching and uncertainty quantification in very high dimensions. Environ. Model. Softw. 109, 191-201. https://doi.org/10.1016/j.envsoft.2018.06.009

Zhang, X., Ellis, R., 2013. Evaluation of Simhyd, Sacramento and GR4J rainfall runoff models in two contrasting Great Barrier Reef catchments, in: MODSIM2013, 20th International Congress on Modelling and Simulation. Modelling and Simulation Society of Australia and New Zealand. Presented at the MODSIM2013, 20th International Congress on Modelling and Simulation. Modelling and Simulation Society of Australia and New Zealand, Adelaide, Australia, pp. 3260-3266. https://doi.org/978-0-9872143-3-1 